Meta

Journal des traducteurs

Translators' Journal

\title{
Traduire In the Night Kitchen, ou de la difficile lecture d'un album
}

\section{Isabelle Nières-Chevrel}

Volume 48, numéro 1-2, mai 2003

Traduction pour les enfants

Translation for children

URI : https://id.erudit.org/iderudit/006964ar

DOI : https://doi.org/10.7202/006964ar

Aller au sommaire du numéro

Éditeur(s)

Les Presses de l'Université de Montréal

ISSN

0026-0452 (imprimé)

1492-1421 (numérique)

Découvrir la revue

Citer cet article

Nières-Chevrel, I. (2003). Traduire In the Night Kitchen, ou de la difficile lecture d'un album. Meta, 48(1-2), 154-164. https://doi.org/10.7202/006964ar
Résumé de l'article

La traduction française de In the Night Kitchen se révèle très décevante. En confrontant cette traduction française et la traduction allemande qui lui est contemporaine, l'étude s'attache à cerner les résistances qu'offre l'album à toute traduction, le mécanisme d'aveuglement qui a présidé à la traduction française et l'écart des possibilités que donne aux traducteurs français et allemand leur langue respective. Elle s'interroge enfin sur la place qu'occupent les images dans la lecture d'un album. Lire un album, c'est lire tout à la fois des images et du texte, même si le traducteur ne rendra compte ensuite que de sa lecture à travers sa traduction du seul texte.
Ce document est protégé par la loi sur le droit d'auteur. L'utilisation des services d’Érudit (y compris la reproduction) est assujettie à sa politique d'utilisation que vous pouvez consulter en ligne.

https://apropos.erudit.org/fr/usagers/politique-dutilisation/ 


\title{
Traduire In the Night Kitchen, ou de la difficile lecture d'un album
}

\author{
ISABELLE NIÈRES-CHEVREL \\ Université de Rennes II, Rennes, France \\ isabelle.nieres-chevrel@uhb.fr
}

\begin{abstract}
RÉSUMÉ
La traduction française de In the Night Kitchen se révèle très décevante. En confrontant cette traduction française et la traduction allemande qui lui est contemporaine, l'étude s'attache à cerner les résistances qu'offre l'album à toute traduction, le mécanisme d'aveuglement qui a présidé à la traduction française et l'écart des possibilités que donne aux traducteurs français et allemand leur langue respective. Elle s'interroge enfin sur la place qu'occupent les images dans la lecture d'un album. Lire un album, c'est lire tout à la fois des images et du texte, même si le traducteur ne rendra compte ensuite que de sa lecture à travers sa traduction du seul texte.
\end{abstract}

\section{ABSTRACT}

The French translation of In the Night Kitchen is a very disappointing one. A comparison of the French and German versions of Maurice Sendak's picture-book demonstrates what could be considered as "untranslatable," what may have led to the French translator's blindness, and how great the discrepancy between German and French is when translating an English text. The paper deals lastly with the part played by pictures in the meaning of a picture-book. To read a picture-book is to read both pictures and text, even if afterwards the translator conveys his whole reading by translating the text only.

\section{MOTS-CLÉS/KEYWORDS}

difficultés de traduction, images, In the Night Kitchen, Sendak, traduction française et allemande

In the month of June

I stand beneath the mystic moon.

E.A. Poe

C'est une déception qui est à l'origine de cette étude. L'anecdote se situe à l'hiver 1972-1973. À l'occasion d'une exposition d'albums contemporains, je découvre la toute récente édition française de l'album de Maurice Sendak, In the Night Kitchen, paru aux États-Unis en 1970, et que Jean-Henri Potier venait de traduire pour les éditions de L'école des loisirs. Je connaissais déjà Max et les Maximonstres (Where the Wild Things Are) album pour lequel j'avais la plus vive admiration ${ }^{1}$. C'est donc avec une certaine gourmandise que je m'isolai pour découvrir ce nouvel album. Après l'avoir lu, je le reposai l'album, déçue et déçue d'être déçue. Je profitai quelque temps plus tard d'un passage à Paris pour me procurer l'album dans sa version originale. Puis, pendant près de deux ans, je lus et relus le livre, fouillant tous ses recoins, m'interrogeant sur les possibles, ou impossibles, solutions d'une traduction française. Je me replaçais donc mutatis mutandis dans la position du traducteur qui doit lire 
l'œuvre originale $=$ construire du sens) pour la traduire, c'est-à-dire tout à la fois imiter des formes et re-produire du sens. Ce n'est que lorsque je crus avoir «épuisé » l'album dans sa version originale (ce qui bien sûr se révéla inexact) que je m'autorisai enfin à me procurer l'édition française. Je compris immédiatement les raisons de ma déception: la traduction française avait totalement fait disparaître ce qui faisait la profondeur de ce livre. Lors de la création de cet album, Sendak écrit à un de ses amis: "It comes from the direct middle of me, and it hurt like hell extracting it. Yes, indeed, very birth-delivary type pains, and it's about as regressed as I imagine I can go. Simply, it's divine ${ }^{2}$.»

Comment une traduction peut-elle en venir à desservir à ce point le texte qu'elle ne pouvait que vouloir servir? Un album pourrait-il poser d'autres problèmes que ces difficultés de traduction bien identifiées que sont les niveaux de langue, le rythme, les jeux de mots éventuels, les références culturelles? Afin de mieux cerner l'échec de la traduction française et pour faire la part entre ce qui relève du traducteur, ce qui est imputable aux contraintes de la langue française et ce qui se dérobe à tout transfert culturel, je me suis procuré une traduction allemande de l'album. Je me propose de confronter l'édition américaine originale, l'édition de L'école des loisirs et l'édition publiée en 1971 à Zurich chez Diogenes Verlag sous le titre In der Nachtküche, dans une traduction signée Hans Manz $z^{3}$.

\section{Une impossible traduction?}

In the Night Kitchen est un album qui fut abondamment traduit et qui offre cependant de grandes résistances à la traduction. Une partie de ces résistances concernent le texte lui-même et elles sont de nature classique. Nous nous trouvons tout d'abord devant un texte fortement assonancé, selon une pratique très fréquente de la littérature enfantine anglo-américaine, mais quasi étrangère à la tradition française. Le récit comporte d'autre part quatre jeux de mots. La déperdition éventuelle ne serait pas catastrophique si ces jeux de mots ne jouaient qu'un rôle marginal dans l'économie du texte. Or il se trouve que ceux-ci sont au cœur de la narration: ce sont eux qui fondent le récit. Le texte nous dit que "The bakers [...] mixed Mickey in batter, chanting: / Milk in the batter! Milk in the batter.. (p. 7-8) . En d'autre termes, les boulangers ont pris Mickey, le jeune héros de l'album tombé dans la pâte à pain, pour du lait [milk], et ils mettent au four un «Mickey-cake»[«milky-cake»] (p. 10). Mickey va donc devoir se libérer de cette confusion qui nie son identité et le réduit à de la pure matière. Il jaillit du four et affirme «I'am not the milk and the milk's not me! I’m Mickey!» (p.12). Le lien entre Mickey et le lait est à nouveau affirmé un peu plus loin, mais sous la forme d'un jeu de mots attribué à Mickey lui-même. L'enfant fait cette fois un jeu de mots au lieu de le subir. Il déclare aux boulangers : «I get milk the Mickey way»(p.19). Au narrateur revient d'expliciter la paronymie: "And he grabbed the cup as he flew up / and up and up / and over the top of the Milky Way in the Night Kitchen.» (p. 20-22). Une ultime paronymie termine l'album, sans que soit donné cette fois le terme qui la sous-tend «[...] Mickey [...] slid down the side / straight into bed / cakefree [ «carefree»] and dried» (p. 31-34). Elle affirme la délivrance de Mickey et souligne le trajet narratif de l'album. Enfin lorsque Mickey chante son bonheur d'être délivré de la confusion «God bless milk and God bless me!» (p. 26), Maurice Sendak lui prête la mémoire d'une Nursery rhyme: 
I see the moon,

And the moon sees me;

God bless the moon,

And God bless me ${ }^{5}$.

Cette référence intertextuelle est nécessairement perdue en traduction, sauf à imaginer une culture qui possède une prière enfantine équivalente ${ }^{6}$.

Le texte de Maurice Sendak est réparti entre trois espaces distincts. La part qui revient au narrateur est imprimée dans des cartouches rectangulaires et cernés, les propos de Michey et des boulangers sont dans des bulles, selon le codage maintenant classique des bandes dessinées. Les traducteurs sont donc contraints par la surface d'impression qui leur est impartie, mais la souplesse du lettrage de Maurice Sendak leur permet d'inscrire dans l'espace imposé un texte soit légèrement plus court soit légèrement plus long. Mais à ces deux premiers types de textes, vient s'ajouter un troisième, discontinu celui-ci et inséré dans l'image elle-même: des noms d'aliments, des marques d'un produit, des prix de vente, des messages publicitaires, des adresses, des numéros de téléphone. On n'en compte pas moins d'une vingtaine dans la grande double page de Mickley surplombant la bouteille de lait (p. 23-24). Ces écritures incluses n'ont été traduites ni dans l'édition allemande ni dans l'édition française. Les éditeurs se sont trouvés devant une difficulté technique propre aux textes illustrés et aux albums, qu'ils connaissent bien et qu'ils résolvent parfois à l'aide de quelques caches. Sans doute ont-ils craint dans ce cas précis de dégrader totalement les illustrations de Maurice Sendak. Or il se trouve que ce troisième niveau de texte n'est pas un simple exotisme, mais qu'il constitue un des espaces de sens du livre. Une partie des inscriptions sont d'ordre privé et elles sont donc aussi opaques pour un lecteur américain que pour un lecteur français ou allemand ${ }^{7}$. Mais d'autres sont parfaitement lisibles pour un enfant de langue anglaise et, comme les cailloux du petit Poucet, elles tracent un chemin dans l'œuvre. On trouve dans la double page 9-10 «Salt», "Cooked. Ready to eat», «Phœnix baking soda», «pure, cream, open » associés sur une même boîte où figure une vache. Le graphisme des boîtes d'aliments de la page 20 mêle texte et images, et l'illustration déploie les étapes - jalonnées de nourriture - de la petite enfance. Une bouteille, sur laquelle la représentation d'un bébé redouble la mention "baby syrop", domine une boîte cylindrique avec le profil d'une tête de vache et les deux inscriptions «condensed food» et «absolutely pure». Devant cette boîte, au premier plan, un des boulangers semble désigner du doigt une seconde boîte cubique où un visage de jeune enfant est associé à trois inscriptions "open» (avec une flèche), «infant food» et «Price $42 \pitchfork »^{8}$. Le doigt prolonge notre regard vers Mickey qui s'envole dans son petit avion et surmonte une quatrième inscription alimentaire sur la facade d'un immeuble «Rolled White Oates. First, Cheapest, Best». Quelle est la meilleure nourriture, la première, la moins chère, celle qui est absolument pure et toujours prête à être consommée? Celle vers lequelle s'élance l'avion de Mickey, le lait de l'enfance. Plonger enfin dans le blanc laiteux de la bouteille de verre, c'est remonter sans doute plus en amont encore, c'est remonter jusqu'au lait maternel et plonger dans l'espace des origines, là où enfin contenant et contenu se confondent. On le voit, c'est toute une dimension du texte, secrète, un peu énigmatique, qui est refusée au lecteur français ou allemand.

La substitution des textes inscrits dans l'image se limite aux onomatopées initiales et terminales. La série initiale «Thump / Dump, Clump, Lump / Bump» devient 
en allemand: «Wumm / Rumm, Pumm, Pumm / Bumm» et en français: «Roum / Doum, Cloum, Ploum / Boum". De manière tout à fait classique, le triomphal cri du coq de Mickey «Cock .a. Doodle Doo!» devient «Kiiii Ke ri Kiiiiii !» en allemand et «Coco Rico!» en français ${ }^{10}$.

La résistance iconotextuelle de In the Night Kitchen à toute traduction va au-delà de ces insertions de la lettre dans l'image. Le prénom américain de Mickey a été choisi par Maurice Sendak comme un discret hommage au héros de Walt Disney (voir note 7). Que le lecteur français ou allemand ne perçoive pas les clins d'œil au graphisme du dessin animé - en particulier dans les deux images circulaires initiale et terminale - n'entrave pas sa lecture. Il identifie par contre aisément les traits de l'acteur Oliver Hardy donnés au trio des boulangers - alors que ceux de Stan Laurel ne sont que fugitivement prêtés à Mickey à la page 13 - parce que les films de ce duo comique sont devenus des classiques internationaux. Au-delà d'un hommage au cinéma de son enfance, Sendak suggère-t-il un «combat» du frêle Mickey-Laurel contre le massif Hardy, dont le triplement confine ici au cauchemar ${ }^{11}$ ? Il n'est pas étonnant qu'une autre culture iconographique américaine - celle de la bande dessinée - soit présente dans cet album. On peut lire en tout petits caractères sur la boîte «Homer's Sugar» de la page 7, «Little Nemo $»^{12}$. Les somptueuses planches de Little Nemo in Slumberland furent découvertes en France grâce à une édition (partiellement en couleurs) publiée par l'éditeur Pierre Horay en $1969^{13}$. Ce fut une révélation. Tout lecteur devenu familier du héros de Winsor McCay était donc prêt à apercevoir trois ans plus tard la ressemblance entre le lit de Little Nemo - représenté de profil avec ses hauts montants de bois - et celui de Cuisine de Nuit. Le lit de Mickey ouvre et ferme l'album à l'instar du lit de Nemo qui ouvre les (premières) planches de Little Nemo et les ferme, à une différence près. La présence du lit à la fin de chaque planche marque chez McCay un violent retour de Nemo au réel, alors que le lit final de Mickey vient accueillir l'enfant pour un sommeil cette fois apaisé. Enfin Maurice Sendak fait deux emprunts au Max et Moritz (1865) de Wilhelm Bush, la séquence narrative de Mickey pris dans la pâte à pain et mis au four, et la croûte de pâte cuite (qui fait à Mickey une combinaison d'aviateur) ${ }^{14}$. La référence est cette fois tout à fait lisible pour un enfant de culture allemande alors qu'elle est invisible en France, où le livre est inconnu. Le maintien ou la perte des allusions intertextuelles et intericonographiques est donc moins fonction de la culture convoquée par l'œuvre originale que des références que culture initiale et culture d'arrivée ont en commun.

Un dernier aspect de In the Night Kitchen se dérobe à la traduction. Il s'agit des jeux entre une forme verbale et une représentation graphique, ce que l'on pourrait appeler des jeux de mots «iconotextuels». Je pense en avoir relevé trois dans l'album. Dans le premier, les deux «termes» du jeu sont présents. Mickey est réveillé par un grand bruit dans la nuit et il crie «Quiet down there!» (p. 2). Le mot engendre la chute: Mickey tombe et tourbillonne dans l'espace. La narration sera toute entière une succession de chutes et d'ascensions jusqu'au repos final de Mickey. Le traducteur allemand conserve le pouvoir de l'adverbe en traduisant «Ruhe da unten!», alors que le traducteur français retient la tournure française la plus attendue «Eh, là-bas! C'est fini?», et introduit du même coup une latéralité qui fait perdre à la protestation de Mickey sa logique d'engendrement graphique. Dans les deux autres exemples, l'iconique est informé par deux expresions idiomatiques qui restent en absence. Les boulangers enfournent Mickey englouti dans la pâte à pain (p. 10). Or, il existe une 
expression anglaise: "to have a bun in the oven» [a bun est un petit pain au lait], dont l'équivalent français pourrait être, dans un registre un peu plus familier, "avoir un polichinelle dans le tiroir». Que l'on rapproche cette expression de la dénomination du four "Mickey Oven», et ce four «devient» le ventre de la mère, le lieu de la genèse de Mickey. Quelques pages plus loin, le narrateur nous dit: "Then Mickey in dough [pâte à pain] was just on his way» (p. 16). Un geste sur la page suivante est à lire comme l'indice d'un jeu de mots dont le second terme n'est pas verbalisé: on voit Mickey faire le salut militaire (p. 17). En américain, a doughboy est un soldat. Mickey serait donc un enfant qui joue à être un soldat en mission. Il affirme un peu plus loin qu'il est «Mickey the pilot» (p. 19). Le narrateur le dira plus modestement «Mickey the milkman» lorsqu'il plonge dans la bouteille de lait (p. 25). De ces trois métiers, seul le dernier figurera dans la version allemande.

\section{Une lecture aveuglée}

La forte résistance de In the Night Kitchen à tout transfert culturel ne suffit pas à expliquer ma déception devant l'édition française de l'album, d'autant que la traduction allemande se révèle tout à fait satisfaisante.

Tout se passe comme si le traducteur français avait été proprement aveuglé par l'écriture assonancée de Maurice Sendak, et qu'il lui avait semblé fondamental de recréer dans le texte français les effets formels du texte américain. Traduire, c'est toujours effectuer des choix. La question est ici la suivante: les choix de Jean-Henri Potier sont-ils pertinents? Le rendement des jeux rimés est-il essentiel dans In the Night Kitchen ${ }^{15}$ ? Je pars d'un exemple. Sendak écrit: «Where the bakers who bake till the dawn so we can have cake in the morn mixed Mickey in batter, chanting» (p. 7). Jean-Henri Potier traduit «Dans le pétrin de la cuisine trois pâtissiers de bonne mine mêlent les œufs et la farine, et chantent. » Pourquoi ajouter cette qualification aux pâtissiers? Pour obtenir en fait trois octosyllabes qui riment:

Dans le pétrin de la cuisine

trois pâtissiers de bonne mine

mêlent les œufs et la farine

Pour privilégier l'imitation d'une forme, le traducteur a été amené à sacrifier deux informations: l'activité nocturne pour le bien commun, à laquelle fait écho la dernière phrase du texte: "And that's why, thanks to Mickey, we have cake every morning» (p. 35), et surtout la présence de Mickey dans la pâte, premier terme nous l'avons vu - de la paronymie qui permet aux boulangers de confondre Mickey et le lait. N'ayant pas noué dès le départ le lien entre Mickey et le lait, le traducteur va se retrouver sans clairvoyance dans les passages stratégiques de l'album. Lorsque Mickey jaillit du four et se libère de la pâte, il déclare aux boulangers: "I'm not the milk and the milk's not me! I'm Mickey!»(p. 12). Dans la traduction française, Mickey dénonce une confusion qui n'a pas été posée ou, plus précisément, il suggère trois confusions possibles: "Je ne suis pas du sucre, ni du lait, ni du beurre. Je suis Mickey. N'ayez pas peur!» Une traduction littérale du passage ne présentait aucune difficulté. Le traducteur français disperce le sens de la protestation de Mickey. On voit ici les ravages qu'a pu entraîné le souci obsédant de la rime. Même remarque et même conclusion pour la chanson libératrice de Mickey dans la bouteille de lait. 
Sendak écrit «I'm in the Milk and the Milk's in Me / God bless Milk and God bless $\mathrm{Me}$ !» (p. 26). De ce Dieu, qui me semble être celui de la Genèse, celui qui nomme les être et les choses et qui garantit à Mickey son identité, il ne reste en français que cette pauvre rime: «Que c'est bon et doux le lait! / Vive le lait quand il est frais!»

L'honnêteté veut de dire que le traducteur français, aurait-il su lire le voyage de Mickey aux sources de son identité, se trouvait de toute manière devant une situation linguistique bien difficile. Le français lui rendait quasiment impossible la re-création des jeux de mots qui donnent à l'album son sens. Le jeu de mot initial sur le prénom du héros est fondateur: sans lui, l'album s'effondre. L'allemand offre à Hans Manz une équivalence simple et pleinement satisfaisante: "Milch / Micky». Aucune solution - une fois que l'on a exclu Pascalet ${ }^{16}$ - ne se présente en français: aucun prénom masculin ne commençe ou se termine par [le]. On ne trouve guère mieux pour les trois autres jeux de mots. Et quelle malchance que l'adjectif correspondant au substantif français en soit phonétiquement si loin «lait / lacté ${ }^{17}$ ! Aucun des deux traducteurs ne rend le jeu de mots de la page 19 («Mickey way»), mais Hans Manz profite cependant de ce que "Milky way» se dise "Milchstrasse» en allemand. Jean-Henri Potier essaie de compenser la perte en rapprochant - quant au sens - les deux termes à la page 22: «plus haut que la voie lactée où le lait coule à volonté», mais le jeu de mots est perdu ainsi que le lien qu'il crée entre le lait et Mickey ${ }^{18}$. Enfin les deux traducteurs ne trouvent aucune solution pour le «cakefree» final. Le seul réel cadeau qu'apporte la langue française est la polysémie du mot «cuisine» qui peut signifier tout à la fois kitchen et cooking. Qu'est-ce qui se cuisine de cette Cuisine de nuit?

Finalement, pour un lecteur français, que raconte Cuisine de nuit? L'essentiel a disparu: la menace qui pèse sur Mickey d'être confondu avec de la matière, l'angoisse de l'identité perdue, la fonction salvatrice de Dieu. Il est remarquable qu'à la page 26 non seulement toute référence à Dieu, mais également toute référence à Mickey ait disparu. Tout se passe comme si le héros n'était pas intimement impliqué dans cette dramatique aventure. Que reste-t-il? L'histoire d'un petit garçon qui, de passif, devient actif et échappe à un trio d'adultes en leur fournissant un des ingrédients dont ils ont besoin pour faire «le pain du matin».

Le traducteur allemand n'est pas arrêté comme le traducteur français par le rendement de l'écriture assonancée de Maurice Sendak. La proximité de ces deux langues accentuées que sont l'anglais et l'allemand va lui permette de créer sans contorsions excessives un texte lui aussi fait "pour l'oreille». Du même coup, son attention reste disponible pour déchiffrer d'autres niveaux du texte.

Là où Jean-Henri Potier peine à faire rimer adjectifs et substantifs, Hans Manz joue en virtuose de tous les temps et de toutes les formes verbales, les fait rimer entre elles ou les associe ici à un substantif, là à un adverbe, comme à loisir. Voici ce qu'il propose pour la page 7, dont le traducteur français avait tant sacrifié le sens dans son désir de rimer: "Hier standen die Bäcker an Tischen, um Micky in Kuchenteig zu mischen, sie schwangen den Teig und sangen." Il fait aussi bien (mieux?) que Sendak décrivant Mickey qui fabrique son avion: "dehnte und bog ihn, formte und zog ihn", quand Sendak avait écrit "He kneaded and punched it and pounded and pulled» (p. 14). Nous avons vu qu'une large parenté des lexiques anglais et allemand offre à Hanz Manz le jeu de mots dont il a besoin «Milch/ Micky». On peut ajouter d'autres proximités lexicales, qui n'ont pas la même importance stratégique, mais qui participent au rythme et à la «couleur» du texte: «bakers/Bäcker», «Milkman/ 
Milchmann", "God/Gott», «bed/Bett» et, bien sûr, "In the Night Kitchen/In der Nachtküche». Hans Manz recrée avec brio les effets d'écriture de Maurice Sendak, sans être condamné à s'écarter des unités de sens du texte original. Il a, de ce fait, toutes chances de ne pas passer à côté de l'essentiel. Micky dénonce la confusion qui le nie: «Ich bin nicht Milch und Milch ist nicht ich, Ich bin Micky!» (p. 12), et il se met lui aussi sous la protection de Dieu «Gott behüte die Milch und behüte mich!» (p. 26). Peut-on être certain pour autant que le traducteur n'a pas simplement suivi un texte anglais qu'il lui était assez facile de transposer, et qu'il a réellement $l u$ l'album de Sendak et construit une interprétation proche de la mienne? Deux indices m'invitent à le penser. Dans la double page 9-10, Hans Manz conserve scrupuleusement la confusion de Mickey, en remontant simplement du gâteau à la pâte («Der MickyTeig» pour "a delicious Mickey-cake») et il introduit dans son texte - cas unique dans tout l'album - la traduction du «Mickey Oven» de l'image: «Der Micky-Teig wurde gehoben und in den Micky-offen geschoben». Le traducteur estime qu'il lui faut donner au moins cette clé au lecteur.

\section{La lecture des images}

Nous savons que toute traduction a tendance à l'explicitation. Sans doute est-ce là le prolongement spontané du travail d'élucidation qui s'effectue dans l'acte de lecture. Les traducteurs connaissent le piège et s'en méfient. Dans le cas précis des traductions d'albums, peut-on entrevoir dans le texte traduit des explicitations de l'illustration originale, qui se trouve, quant à elle, conservée dans l'édition étrangère? En d'autres termes, arrive-t-il au traducteur de verbaliser une information qui n'est donnée que par l'image? Dans la traduction allemande, il me semble avoir rencontré par trois fois ce mécanisme, mais toujours dans un souci de rythme ou de rime. Pour rendre «Past the moon \& his mama \& papa sleeping tight» (p. 4), Hans Manz utilise une information que seule l'image lui a donnée "Am Vollmond vorbeizog und an Papa und Mama». Lorsque Mickey remonte du fond de la bouteille pour verser du lait aux boulangers, le traducteur exploite un détail de l'image pour avoir sa rime «Er schwamm nach oben, goss Milch vom Rand hinunter, wo der Kuchenteig stand» (p.27). De même, la dernière illustration donne au traducteur sa clausule et sa rime: « [Micky] glitt hinunter sehr tief / sprang in sein Bett / war trocken, schlief» (p. 34). Dans le texte français, les explicitations sont plus abondantes. La clausule du traducteur français est proche de celle du traducteur allemand, mais en choisissant «Et, bien au chaud, il se rendort», elle souligne la structure globale de l'album ${ }^{19}$. À deux reprises, la présence des illustrations introduit par analogie l'univers du jeu enfantin, lors de la fabrication de l'avion ("Comme de la pâte à modeler, il la pétrit et la travaille» p.14) et lors la glissade le long de la bouteille («Puis il descend en toboggan» p. 32). L'explicitation des images peut entraîner des inflexions de sens. JeanHenri Potier inverse les regards en traduisant: «Madame la lune le voit, mais ni maman, ni papa.» (p. 4), alors que c'est Mickey (qui exclame «OOH») et nousmêmes qui voyons la lune - même s'il est vrai que la lune veille tout au long sur Mickey. Il interprète les expressions des boulangers (qui sont pour lui des pâtissiers) comme des expressions de peur («Je suis Mickey. N'ayez pas peur!»p. 12), puis d'affolement («Les trois pâtissiers affolés» p. 17), là où on pourrait ne lire qu'une réaction de surprise, puis de colère (on a «howling» dans le texte original ). Dans le 
premier exemple, Jean-Henri Potier déplace la peur de l'enfant sur le trio des adultes et anticipe l'inversion des rapports de force. Dans le second, l'affolement n'est pas si mal venu parce qu'il suggère une vulnérabilité des pâtissiers et nous prépare à ce ton de grande personne que prend Mickey à la page suivante en s'exclamant: "Vous voulez du lait, mes garçons» (p. 19) [ "What's all the fuss?» dit le texte de Sendak].

Quant à lire les images, le traducteur français aurait-il pu trouver dans celles de Maurice Sendak quelques détails qui l'auraient guidé dans le déchiffrement du texte et dans l'interprétation de l'album? Il y a deux couples d'indices dans ce que Sendak dessine mais ne verbalise pas. Les premiers sont de nature religieuse et renvoient à la culture juive. Lorsque Mickey termine sa chute dans la pâte à pain, on voit apparaître sur la page de droite trois boulangers: le premier porte un sac de farine, le second une boîte de sel, le troisième une boîte - dont le contenu est difficilement lisible - est sans doute «baking soda $»^{20}$. La boîte de sel est ornée d'une étoile de David (p. 6). On retrouve la boîte et son étoile dans le coin gauche de la page 9, quand l'un des boulangers s'avance avec une certaine solennité pour enfourner le «Mickey-cake». Lorsque Mickey jaillit du four, la pâte lui fait comme un habit et le fragment qu'il porte sur la tête fait office de petite kippa juive ${ }^{21}$.

Ces deux signes auraient dû conduire le traducteur français à prendre très au sérieux la prière que Mickey adresse à Dieu, de même que le second couple d'indices - une bouteille et un four - qui nous "parlent» de mort et de résurrection. La boîte de «baking soda» de la page 6 est remplacée sur la page suivante par une bouteille jaune, posée par terre près de trois coquilles d'œuf, sur laquelle on peut lire «Phœnix baking soda». On est passé de la chimie au mythe. Cette bouteille apparaît par trois fois et elle accompagne Mickey dans son épreuve. Dans cette page 7, seule la tête de Mickey émerge de la pâte; sur la page en vis-à-vis, il ne reste plus que sa main qui s'agite (un appel au secours?). Mais la bouteille est là, comme une promesse. Dans la double page suivante, sont alignées de gauche à droite la boîte de sel avec son étoile, la bouteille toujours accompagnée d'une coquille d'œuf et la porte du four ouverte, prête à «recevoir» le «Mickey-cake». Dans une troisième et dernière étape, Mickey jaillit du four et de la pâte: la vapeur masque tous les espaces d'écriture, et seules demeurent, encadrant Mickey, «[P]oenix [b]aking soda» et «Mickey oven». Ce four qui porte le nom de Mickey autorise deux lectures, qui ne sont pas incompatibles. La première est individuelle: le four est une des étapes de cette re-création et résurrection de Mickey, à laquelle nous pouvons associer la discrète référence à la propre résurrection de Maurice Sendak à l'hôpital anglais de Gateshead (voir note 7). La seconde est collective: Maurice Sendak sait qu'il y eut des fours qui ne furent pas des fours de fiction, des rêves dont les petits héros peuvent ressortir pour faire triompher leurs forces de vie. Ce qui n'est ici qu'un four "pour de faux» affleurera - mais sans jamais ne se dire - vingt ans plus tard dans Dear Mili (1988), puis dans We are all in the dump with Jack and Guy (1993), avec la silhouette du mirador d'un camp de concentration.

Lire ces quatre indices dans l'image imposait de reconnaître à Mickey des préoccupations profondes, excluait de réduire sa prière à un simple éloge du lait frais et invitait le traducteur à explorer d'autres voies, à tenter d'autres choix.

Les résistances à la traduction de In the Night Kitchen sont infiniment supérieures à celles que l'on rencontre d'ordinaire dans les albums pour enfants. Les éditions françaises et allemandes de l'album de Maurice Sendak me semblent mettre en lumière quatre problèmes. 
J'ai longtemps pensé que le médiocre rendement français des albums pour enfants anglais, allemands, suédois dont le texte original était fortement rythmé et rimé, voire écrit en vers, résultait pour l'essentiel de l'absence d'une tradition similaire dans la culture française. Mais ce travail sur Sendak me conduit à nuancer ma position et à estimer qu'il faut tout autant prendre en compte les spécificités de la langue française. Sans doute la culture enfantine française a-t-elle rompu dès son émergence au $\mathrm{XVIII}^{\mathrm{e}}$ siècle avec le substrat populaire de nos comptines et formulettes. Mais ces petits vers régis par le mètre et la rime n'ont jamais eu la richesse rythmique et musicale des nursery rhymes anglaises par exemple. Ajoutons que la littérature pour la jeunesse $\mathrm{du} \mathrm{XIX}^{\mathrm{e}}$ siècle n'a rien produit en France qui ressemble à la littérature anglaise de nonsense, elle-même nourrie de la tradition des nursery rhymes. Est-ce à dire qu'il est impossible de rendre en français des pans entiers de la littérature enfantine étrangère? Non, mais il y faut beaucoup d'art. On ne peut que saluer la réussite de Marie Farré à traduire Algernoon and other Cautionary Tales de Hilaire Belloc et celle de Christian Poslaniec à adapter The big brag du Dr Seuss ${ }^{22}$.

Lorsque les albums sont traduits dans une langue étrangère, nous avons pour habitude de considérer que les images, échappant à la malédiction de Babel, ne posent de problème dans la culture d'arrivée qu'en fonction de leur dimension référentielle. In the Night Kitchen nous rappelle que les images peuvent être nées d'expressions parfois prises au pied de la lettre. Si l'expression est présente dans le texte original, le travail du traducteur relève alors de la résolution d'un jeu de mots, pour laquelle on ne pourra jouer que sur l'un des deux termes. Si l'expression est absente et qu'il est exclu de toucher à l'image, la déperdition semble inéluctable ${ }^{23}$.

À ce problème relativement classique (les traducteurs de Through the lookingglass l'ont rencontré avec «les insectes du miroir» représentés par John Tenniel), l'album contemporain ajoute une difficulté supplémentaire, celle des textes insérés dans l'image. Sendak se situe encore dans une apparente analogie au monde réel: écritures sur les boîtes d'aliments, publicités sur les facades des immeubles. Mais d'autres artistes, plus proches de nous, vont systématiquement jouer de la confusion $\mathrm{du}$ lisible et $\mathrm{du}$ visible dans des images sans profondeur, pratiquant le collage avec des fragments de journaux, conviant dans une même page - comme le fait Béatrice Poncelet - Le Petit Poucet illustré par Gustave Doré, Maria des mers illustré par Ivan Bilibine, Max und Moritz de Wilhelm Busch, Le dirigeable cage-à-mouches numéro un de O'Galop, dont l'un des deux héros est remplacé... par le Mickey de In the Night Kitchen $^{24}$. Il y a dans ces collages et ces montages un nouveaul type de résistance à la traduction.

Le dernier problème est à mes yeux le plus fondamental. Obsédé par un problème de traduction, Jean-Henri Potier n'a pas pris le temps de lire In the Night Kitchen. Ceci pose une question essentielle: qu'est-ce que lire un album? Lire un album, ce n'est pas lire un texte, c'est lire un livre; c'est lire ces entrelacs de sens que construisent le texte et les images. Il restera ensuite au traducteur à rendre la richesse de sa lecture par la force de sa seule traduction du texte.

\section{NOTES}

1. Where the wild things are est paru aux États-Unis en 1963 et il a été publié en 1967 chez Delpire sous le titre Max et les maximonstres, avant d'être repris à L'école des loisirs.

2. Cité par Selma G. Lanes, 1980, The Art of Maurice Sendak, New York, Harry N. Abrams, page 174. 
3. Le nom du traducteur figure en bonne place dans la page titre intérieure où il a été inséré selon le même lettrage, alors que celui du traducteur français ne figure qu'en petits caractères dans les informations éditoriales obligatoires.

4. L'album n'est pas paginé. Je numérote les pages à partir du début du texte de Sendak, la page impaire se trouve donc du même coup être celle de gauche. Les barres inclinées marquent le passage du texte d'une bulle ou d'une page à la suivante.

5. Iona and Peter Opie, 1975, The Oxford Dictionary of Nursery Rhymes, Oxford, O.U.P., p. 312.

6. L'inévitable déperdition se retrouve dans un autre album de Sendak, Higglety Pigglety Pop! (1967) traduit en français en 1980 sous le titre Turlututu Chapeau Pointu! La combinaison narrative de deux nursery rhymes fonde deux autres albums de Maurice Sendak, Hector Protector (1965) et We are all in the dumps with Jack and Guy (1993). Aucune culture enfantine «sous-entendue» ne fonctionne alors pour un lecteur français.

7. «Kneitel's Fandango» (p. 5) = Kenny Kneitel, collectionneur de Mickey Mouse; le nom de la chienne de Sendak, Jennie et sa date de naissance, 1953 (sac du boulanger de gauche, p. 7) et sur un sac à terre - presque illisible - «Killingworth, Connecticut», son lieu de naissance; entre les doigts du boulanger de droite, un portrait de la mère de Sendak. Au centre de la double page 9-10, deux adresses d'enfance de Sendak à New-York, qui nous donne également sa date de naissance à la page 15. Dans la grande double page 23-24, «Schickel» est un hommage à Richard Schickel, auteur d'une biographie de Walt Disney. Enfin page 32, l'inscription sur un bâtiment «Q.E. Gateshead» renvoie à l'hôpital anglais où Maurice Sendak fut soigné durant l'été 1967 d'une attaque cardiaque. Toutes ces éclaircissements sont donnés par Selma G. Lanes (op. cit.) dans le chapitre qu'elle consacre à In the Night Kitchen.

8. Né en 1928, Maurice Sendak a 42 ans lors de la publication de l'album.

9. Les hasards de la langue font que le traducteur allemand peut conserver telle quelle la bulle «Mama, Papa» de la page de dédicace et de la page 4. La version française ajoute discrètement un « $\mathrm{m}$ » pour obtenir «maman».

10. Mais il reste surprenant que deux éditeurs aussi sérieux que L'école des loisirs et Diogenes Verlag ne respectent pas le lettrage rouge choisi par Sendak et lui substituent un noir opaque en allemand et un lettrage noir évidé en français.

11. Maurice Sendak dit que cette invention des trois boulangers renvoie à un souvenir d'enfance, à une publicité pour les «Sunshire Bakers» représentant trois gros boulangers et accompagnée du slogan "We Bake While You Sleep». C'est la diffusion de la télévision d'un film de Laurel et Hardy Nothing but trouble - qui lui donna l'idée de leur donner le physique de Hardy. ( Selma G. Lanes, op. cit. p. 174 et 179).

12. Maurice Sendak dit avoir découvert l'œuvre de Winsor McCay, dont il ignorait tout jusqu'alors, à l'occasion d'un exposition au Metropolitan Museum of Art en 1965, qui présentait des planches de Little Nemo in Slumberland. "I now sent me scooting back, with new eyes, to the popular art of my own childhood» ( Selma G. Lanes, op. cit. p. 175).

13. J'ignore ce qu'il en est d'éventuelles éditions allemandes.

14. On peut se demander si l'idée soudaine de Maurice Sendak de prêter aux boulangers la silhouette et le visage de Hardy ne trouve pas son fondement dans une réminiscence inconsciente du profil et de la silhouette - massive et blanche - du boulanger de Wilhelm Busch. L'appropriation personnelle des deux héros de Bush par 'Moritz' Sendak se manifeste en 1963 lorsqu'il donne au héros de Where the Wild Things Are le prénom de Max.

15. Ce qu'il est par exemple dans les traductions des albums du Dr Seuss.

16. Héros de l'écrivain Henri Bosco.

17. On en regrette d'autant plus que le traducteur ait négligé de traduire «Mickey the milkman» (p. 25). La profession de «laitier» gardait cette fois toute la lisibilité de son radical.

18. Dans un long article intitulé «La cuisine de Maurice Sendak», Pierre-Pascal Furth suggère que cette dernière traduction fait écho au passage d'Exode (III, 8) qui évoque «une contrée où ruisellent lait et miel» (Europe, novembre-décembre 1979, Paris, nº 607-608, p. 82).

19. Tout ce récit de rêve est rendu au présent par le traducteur. Le prétérit est une source de difficultés en français, et le présent du texte rejoint ici le "présent» des images: le rêve de Mickey comme si vous y étiez.

20. C'est-à-dire du bicarbonate de soude; on en met une pincée pour fabriquer de nombreux pains.

21. Maurice Sendak appartient à une famille juive d'origine polonaise.

22. Philibert (illustrations de Quentin Blake), 1991, Paris, Gallimard; Le plus vantard, 1986, Paris, L'école des loisirs. 
23. Edmond Cary est, à ma connaissance, le premier spécialiste des problèmes de traduction à avoir évoqué les problèmes posés par la traduction des livres pour enfants. Dans un cours radiodiffusé en 1958, il évoque la tyrannie des illustrations: «Le texte anglais parle de «traffic jam» (embouteillage) et joue sur le mot «jam», qui signifie aussi confiture. Le traducteur aurait la partie belle avec les doubles sens du mot embouteillage, avec les voitures en carafe, que sais-je encore. Mais l'image montre, d'une part des autos et, de l'autre, un magnifique pot de marmelade» (Edmond Cary, 1985, Comment faut-il traduire? Lille, Presses Universitaires de Lille, p. 54).

24. Chez eux, chez elle ou chez elle, 1997, Paris, Éditions du Seuil. Précisons que toutes ces citations iconotextuelles sont retravaillées par l'artiste. 